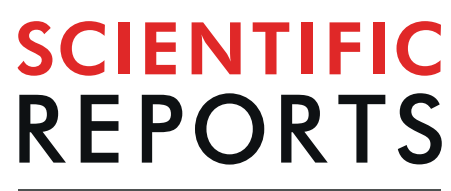

\title{
Single variable domains from the $T$ cell receptor $\beta$ chain function as mono- and bifunctional CARs and TCRs
}

Julyun $\mathrm{Oh}^{1}$, Dora Toledo Warshaviak ${ }^{1}$, Mikayel Mkrtichyan ${ }^{1}$, Melanie Lisette Munguia ${ }^{1}$, Abby Lin ${ }^{2}$, Falene Chai ${ }^{2}$, Craig Pigott $\mathbb{D}^{2}$, Jaspal Kang ${ }^{2}$, Michael Gallo² \& Alexander Kamb ${ }^{1 *}$

Cell therapy using T cell receptors (TCRs) and chimeric antigen receptors (CARs) represents a new wave of immunotherapies garnering considerable attention and investment. Further progress in this area of medicine depends in part on improving the functional capabilities of the engineered components, while maintaining the overall size of recombinant constructs to ensure their compatibility with existing gene delivery vehicles. We describe a single-variable-domain TCR (svd TCR) that utilizes only the variable domain of the $\beta$ chain (V $\beta$ ). This V $\beta$ module not only works in TCR and CAR formats, but also can be used to create single-chain bispecific CARs and TCRs. Comparison of individual ligand-binding V $\beta$ domains in different formats suggests that the lone $\mathrm{V} \beta$ sequence controls the sensitivity and a major part of the specificity of the CAR or TCR construct, regardless of signaling format, in Jurkat and primary T cells.

Cell therapy promises to revolutionize certain aspects of medicine, and has recently achieved a significant milestone with approval of the first engineered chimeric antigen receptor T cells (CAR-Ts) ${ }^{1,2}$. This therapeutic modality depends on receptor molecules, typically engineered antibody single-chain variable fragment (scFv) domains, to redirect $\mathrm{T}$ cell activity to specific cell surface target antigens expressed on the cancer cell. Because recurrently expressed, tumor-selective surface proteins are extremely rare among solid tumors, drug discovery has recently begun to explore the utility of peptide-MHC complexes (pMHCs) as target opportunities for CAR-Ts and T-cells with engineered receptors or native TCRs.

pMHCs are the natural ligands for TCRs and as such lend themselves to targeting by TCRs and their derivatives $^{3}$. Class I MHC molecules consist of a molecular dimer of an $\alpha$ chain associated with $\beta 2$ microglobulin. This dimer binds in a degenerate manner to individual short peptides while folding in the endoplasmic reticulum to create the pMHC complex ${ }^{4}$. The $\mathrm{pMHC}$ is then transported to the membrane surface where it can be recognized by cognate T cells. If a certain binding threshold is crossed, pMHCs can trigger activation and effector function of T cells through TCRs.

$\mathrm{T}$ cell receptor heterodimers $(\alpha \beta)$ are expressed on T cell surface in complex with the CD3 proteins, which provide the necessary signaling domains in the stoichiometry $\alpha: \beta: \gamma: \delta: 2 \varepsilon: 2 \zeta^{5}$. The $\alpha$ and $\beta$ subunits form the central pMHC binding element, which has significant structural similarity to its relative, the antibody antigen-binding fragment (Fab). Variable domains of the $\alpha$ and $\beta$ subunits are assembled from discrete $V, D$, and J gene elements via genomic recombination. The $\beta$ chains are inherently more complex than the $\alpha$ chains since they include $\mathrm{D}$ regions and two junctional regions (V-D and D-J), whereas $\alpha$ chains just have one (V-J). Both the $\alpha$ and $\beta$ subunits typically contribute to binding a small region on the class I MHC ligand distal to the membrane that includes residues from the MHC molecule as well as the short peptide complexed with it ${ }^{6,7}$.

pMHCs afford the option to target peptides derived from intracellular proteins that reside in the cytoplasm and are otherwise inaccessible to large molecules, such as monoclonal antibodies. As more elaborate cell therapeutics are contemplated, designed and deployed, the small size and modularity of the component parts become increasingly valuable. For example, the creation of bifunctional receptors, i.e., receptors with two linked domains that bind different ligands, affords the option to target two different antigens at once, a potentially very valuable asset in certain therapeutic settings. However, some aspects of TCRs may limit their utility as modular 
components of large-molecule and cell-therapy applications, constraints potentially rooted in their basic structure and function. Such structural complexity and increased size may hamper the practical development of next-generation bifunctional therapeutics.

The appeal of bifunctional targeting molecules, coupled with packaging limitations of gene-transfer vectors such as lenti- and retroviruses, raises the question of whether TCRs can function as a single binding domain; e.g., as a V $\beta$ devoid of its normal partner, the $\alpha$ variable domain $(\mathrm{V} \alpha)$. To our knowledge, no instance of such a streamlined TCR has been reported, though shark species have a variety of novel TCRs that are distinct from mammalian orthologs ${ }^{8,9}$. If such a simplified TCR variable domain that retains specificity and functional activity against particular pMHCs were possible, it could open a path to engineering smaller, more complex binding capabilities with svd TCRs.

Though svd TCRs are an attractive concept, it is not evident that such stripped-down binding domains can be derived easily from mammalian TCRs. The three-dimensional structure of TCRs reveals a complex where the TCR V $\alpha$ and $\beta$ chains pack together, covering a large $\left(>200 \AA^{2}\right)$ hydrophobic surface. Removal of the $V \alpha$ domain exposes this surface to destabilizing solvent interactions. By analogy with protein engineering efforts on antibodies conducted over the past decades, such dramatic changes in structure require significant adjustments, either by design or selection, to accommodate the single-variable domain format ${ }^{10,11}$. Despite the challenges, antibody $\mathrm{VH}$-only domains are increasingly popular for generation of innovative clinical molecules with multi-specific binding properties ${ }^{12,13}$.

Here we demonstrate that svd TCRs can be constructed from a V $\beta$ domain with no additional sequence engineering within the framework and constant regions of the $\beta$ chain. These svd TCRs express stably on the surface of mammalian cells, including T cells. They bind pMHC tetramers selectively and appear to trigger T cells in much the same manner as full TCRs. Moreover, they function in tandem as CAR and TCR bifunctional proteins. Such V $\beta$-only domains provide a powerful basis for next-generation engineered $\mathrm{T}$ cell therapeutics and shed light on the mechanisms that dictate signaling sensitivity of TCRs and CARs.

\section{Results}

Identification of $\beta$-only pMHC binding domains. To identify peptide-specific pMHC-binding molecules, $\mathrm{V}(\mathrm{D}) \mathrm{J}$ recombination in cell culture was used to generate sequence diversity in the TCR V $\beta$ domain while keeping the V $\alpha$ constant in a controlled fashion in HEK293 cells (see patent WO2017/091905 for details about the HuTARG ${ }^{\mathrm{TM}}$ platform used to generate the TCR diversity). Over 100 million TCR-expressing cells were sorted for TCRs specific to the HLA-A*02-01 allele in complex with NY-ESO-1 $9 \mathrm{~V}$ variant peptide (SLLMWITQV) or MAGE-A3 peptide (FLWGPRALV) using pMHC multivalent tetramers. Note that C-terminal to the C $\alpha / \beta$ domains, the library construct encoded the human $\mathrm{CD} 3 \zeta$ transmembrane and cytoplasmic domains fused to the $\beta$ chain to facilitate its expression in HEK293 cells, and subsequent analysis. Specific pMHC-binding cells were enriched over multiple rounds and purified from the library. During the process, $\alpha$ chain expression was inadvertently lost and $\beta$ chains that endow specific epitope-binding in the absence of a second TCR variable domain were recovered. Such $\beta$-only clones were used as the basis of the studies described below.

$\beta$-only domains express stably and show selective PMHC binding in CAR and TCR formats. Several clones of $\beta$-only domains selective for either HLA-A2/NY-ESO-1 peptide or HLA-A2/MAGE-A3 peptide were recovered from the HuTARG ${ }^{\mathrm{TM}}$ sort and sequenced. Among these, 3 and 4 unique CDR3 sequences were identified for NY-ESO-1- and MAGE-A3-selective binders, respectively (Supplementary Table S1). Interestingly, all $7 \beta$ idiotypes for both pMHC targets utilize the TRBV5-8*01 segment, suggesting that there are structural properties of this $\mathrm{V} \beta$ segment that facilitate stability of a single domain in the absence of its normal $\mathrm{V} \alpha$ partner (see below). Furthermore, the average CDR3 loop length was 16.3 amino acids (range 12-20), 2 amino acids longer than the average observed among a set of human TCR V $\beta$ sequences ${ }^{14}$. These idiotypes were characterized for their ability to express in HEK293T cells and bind pMHC targets by flow cytometry. The TCR $\beta$ F1 (8A3) antibody was used to detect surface-expressing TCR $\beta$ chains. This antibody does not stain the native form of the $\mathrm{TCR}^{15}$, allowing detection of the TCR $\beta$ chain in CAR formats only. pMHC tetramers were generated by conjugating biotin-labeled, peptide-containing MHC Class I A*02:01 with fluorophore-labeled streptavidin in a tetramer format. All idiotypes were expressed stably on the cell surface with various tetramer-binding abilities (Supplementary Fig. S1). The strongest binders, idiotype \#2 for a NY-ESO-1-targeted V $\beta$-only domain and idiotype \#5 for a MAGE-A3-targeted domain, were selected for further studies.

To assess the specificity and versatility of these V $\beta$-only domains, the NY-ESO-1- and MAGE-A3-binding idiotypes (\#2 and \#5, respectively) were formatted as TCR $\alpha \beta$ s (referred to as svd TCR) and CARs (referred to as $\mathrm{V} \beta$-only CAR). For the TCR $\alpha \beta$ format, a generic $\alpha$ chain that is abundantly expressed in humans was used as a surrogate (TRAV $41 * 01 / \mathrm{J} 49 * 01$ ). For the CAR format, $\mathrm{V} \beta$ s were linked to the $\beta$ chain constant region (C $\beta$ ), which in turn was fused to a $\mathrm{CD} 3 \zeta$ transmembrane and intracellular signaling domain (first-generation CAR) or a CD28 transmembrane region and CD28 and CD3 $\zeta$ intracellular signaling domains (second-generation CAR) (Fig. 1a). For comparison we used the previously reported NY-ESO-1-targeted TCR clone $1 \mathrm{G} 4^{16}$ and MAGE-A3-targeted mouse $\mathrm{TCR}^{17}$, as well as CARs with scFvs derived from reformatted antibody surface display (HuTARG ${ }^{\mathrm{TM}}$ ) libraries. These constructs were transfected into HEK293T cells to assess expression and binding specificity by flow cytometry. Mixtures of 7 off-target pMHCs with different HLA allele and unrelated peptides were used to generate negative-control tetramer. Flow cytometry data showed that both NY-ESO-1- and MAGE-A3-targeted V $\beta$-only CARs are stably expressed and selectively bind to target tetramers in HEK293T cells. svd TCRs also express and show specificity for the pMHC target, but with significantly lower binding (Fig. 1b).

To examine expression and function of V $\beta$-only CARs and svd TCRs in a more natural setting, the constructs were transfected into the Jurkat human T cell line. Additional CAR constructs with the V $\beta$-C $\beta$ domains linked to $\mathrm{CD} 28$ transmembrane region with $\mathrm{CD} 28,4-1 \mathrm{BB}$ and $\mathrm{CD} 3 \zeta$ intracellular signaling domains (third-generation CAR) 
a

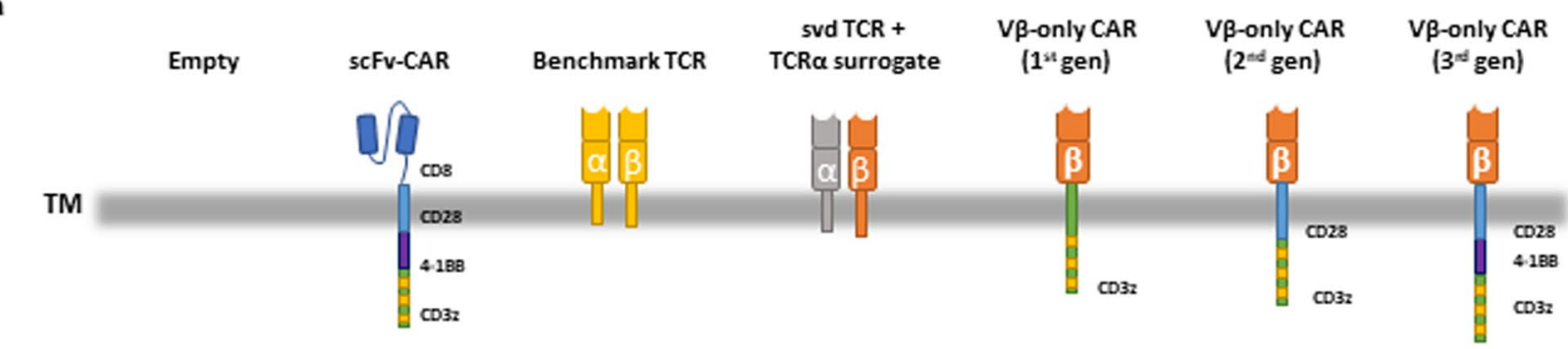

b
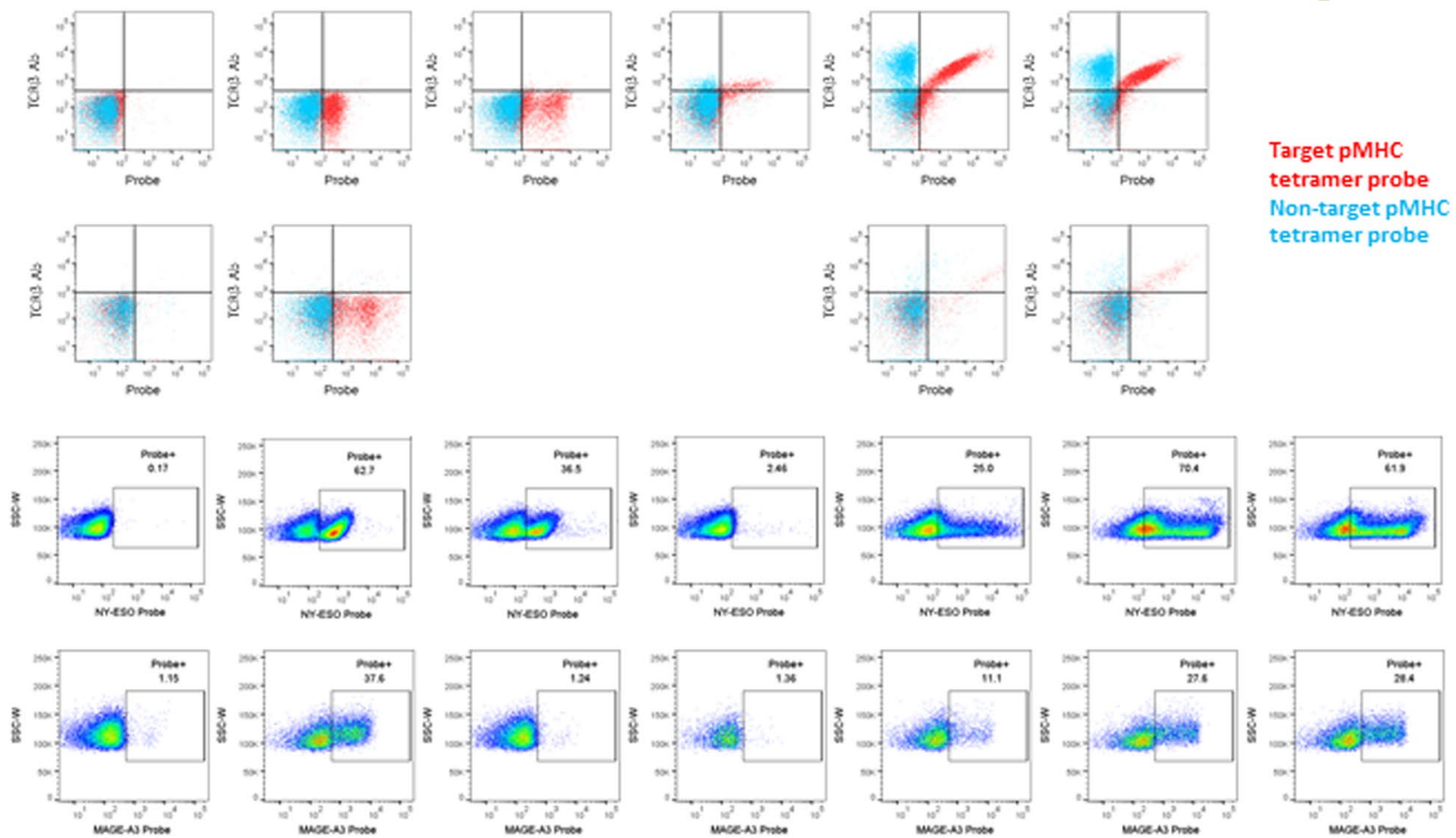

Figure 1. V $\beta$-only domains in CAR formats express stably and show selective pMHC binding. (a) Schematic of the constructs used in the experiments. Note that the $1^{\text {st }}$ gen $\mathrm{V} \beta$-only CAR will dimerize through CD3 $\zeta$ TM, which is not depicted in the schematics. (b) FACS plot of HEK293T cells transfected with NY-ESO-1 (top) and MAGE-A3 (bottom) pMHC-targeted constructs. The $\mathrm{x}$-axis reflects the amount of target (red) and non-target (blue) pMHC tetramer bound to cell. The y-axis reflects the amount of the TCR $\beta$ chain on the cell surface. (c) FACS plot of Jurkat cells transfected with NY-ESO-1 (top) and MAGE-A3 (bottom) pMHC-targeted constructs. The $\mathrm{x}$-axis reflects the amount of target $\mathrm{pMHC}$ tetramers bound to cell.

were also tested. All NY-ESO-1- and MAGE-A3-targeted V $\beta$-only CARs showed detectable tetramer binding in Jurkat cells. Slightly higher tetramer binding percentages were observed in second- and third-generation CARs compared to first-generation. However, svd TCR did not bind tetramer for either pMHC target (Fig. 1c).

The TRB5-8 V $\beta$ segment encodes a sequence that may more readily form a single functional binding domain. Based on the crystal structure of a V $\beta-5$ TCR complexed with MAGE-A3 pMHC, we modeled one of the MAGE-A3 V $\beta$-only domains described here (idiotype \#5) and the results matched the expectation that some adjustments to the polypeptide backbone are required (Fig. 2a) ${ }^{18}$. A molecular dynamics simulation of the TRB5-8 V $\beta$-only structure revealed that the interface with $\mathrm{V} \alpha$ is at least partly covered by a collapse of the CDR3 loop over its hydrophobic surface to accommodate the loss of $V \alpha$ (Fig. 2b). In addition, modeling two other TCRs (6AT6 and 6AVG) ${ }^{19}$ that contain V segments from other families, suggests that at least some other $\mathrm{V} \beta$ domains require more radical changes to form a thermodynamically stable structure absent the $\mathrm{V} \alpha$ domain, compared to V $\beta 5-8$ (Fig. 2c,d). Specifically, the crystal structure of a TRBV28-encoded domain shows nearly $80 \%$ more hydrophobic surface area exposed, compared to the V $35-8$-only domain. Presumably such sequences have a more challenging route to formation of a stable single globular domain.

V $\beta$-only domains in CAR formats are functionally active. To assess whether target binding by $\mathrm{V} \beta$-only domains can lead to functional activity, engineered Jurkat cells that expressed an NFAT-driven luciferase reporter gene were transfected with V $\beta$-only constructs. Serially diluted NY-ESO-1 and MAGE-A3 peptides were loaded onto T2 cells (HLA-A2+) 16 hours prior to co-culturing with transfected Jurkat effector cells. Population-averaged NFAT signal was measured by luminescence after 6 hours of co-culture. Peptide-titration 
a

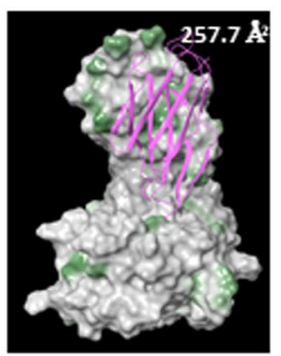

b

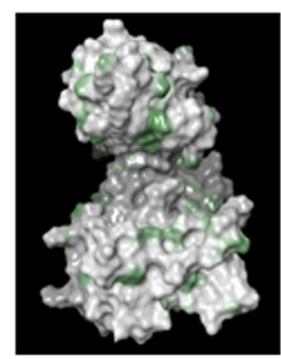

c

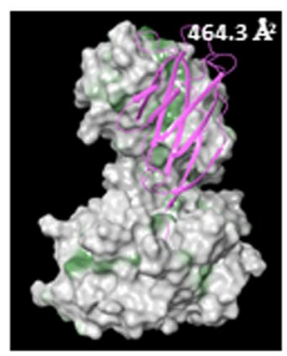

d

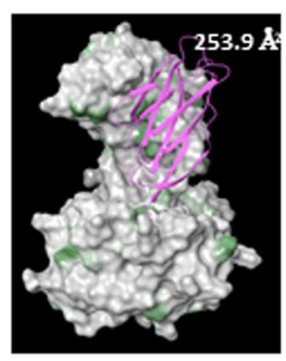

Figure 2. Structural homology modeling analysis. For (a-d) homology models that use the atomic coordinates of the MAGE peptide/HLA-A2 pMHC in complex with a VR5 TCR (5BRZ). Hydrophobic patches on the spacefilling surface model are labeled green; charged and neutral surfaces white. For A, C and D, a ribbon cartoon shows where $\mathrm{V} \alpha$ normally binds to $\mathrm{V} \beta$ in the TCR $\alpha / \beta$ dimer. The intersection between the hydrophobic surface and the $\alpha / \beta$ contact surface was calculated and is shown in $\AA^{2}$. (a) MAGE-A3 svd TCR homology modeled on 5BRZ template without the TCR $\alpha$ subunit. (b) MAGE-A3 svd TCR homology modeled on 5BRZ template after 100 nsec molecular dynamics simulation. (c) TRBV28 domain from previously crystalized TCR (6AT6) homology model on 5BRZ template without the TCR $\alpha$ subunit. (d) TRBV9 domain from previously crystalized TCR (6AVG) homology model on 5BRZ template without the TCR $\alpha$ subunit.
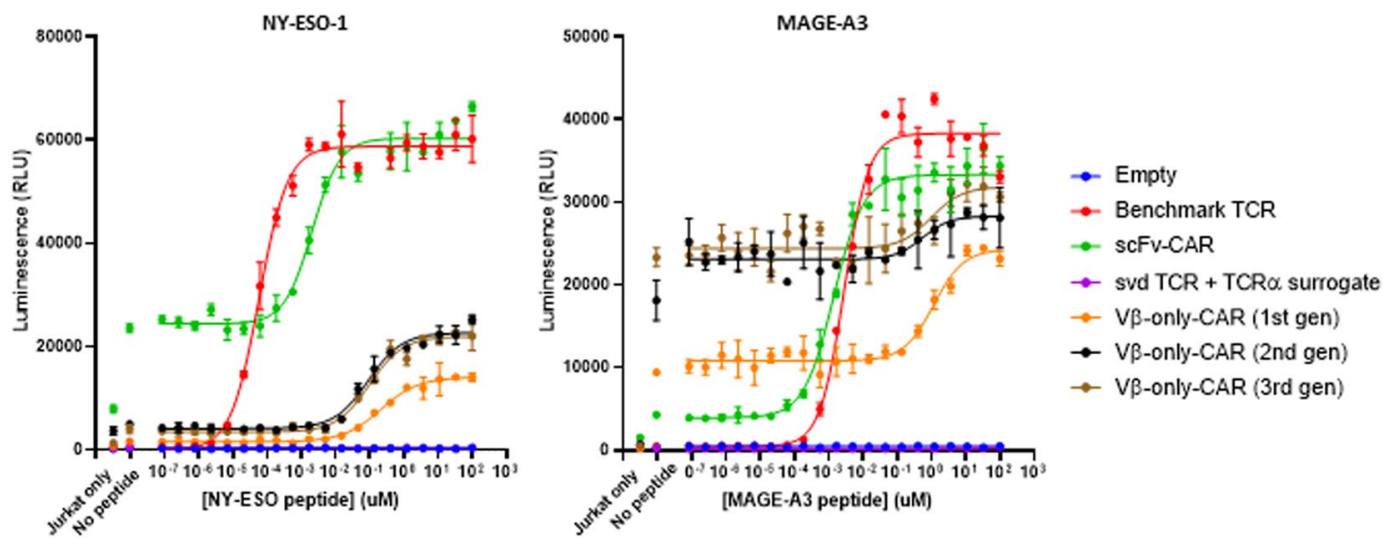

Figure 3. V $\beta$-only domains in CAR formats show target peptide-specific functional activation. NFAT-luciferase signal of transfected Jurkat cells after $6 \mathrm{hrs}$ of co-culture with NY-ESO-1 (left) or MAGE-A3 (right) peptideloaded T2 cells. The error bars indicate SD $(n=2)$.

curves showed that both NY-ESO-1 and MAGE-A3 V $\beta$-only CARs trigger peptide-concentration-dependent NFAT activation (Fig. 3). The activation is specific to its respective target peptides (Supplementary Fig. S2). First-generation CAR formats showed about two-fold less sensitivity than second- and third-generation CARs with both binders based on the $\mathrm{EC}_{50}$ (Supplementary Table S2). Compared to control third-generation CARs with affinity-matured scFv binders, third-generation CARs with $\mathrm{V} \beta$ domains were $\sim 100 \times(\mathrm{NY}$-ESO-1) and $\sim 1000 \times($ MAGE-A3) less sensitive (Supplementary Table S2).

The MAGE-A3 V $\beta$-only CARs showed elevated background NFAT signaling (Fig. 3). However, this feature does not appear to be inherent to all V $\beta$-only CAR constructs as the other three idiotypes of MAGE-A3 V $\beta$-only modules showed lower tonic signal than the scFv CAR (Supplementary Fig. S3). To explore the cellular localization and aggregation profile of the $\mathrm{V} \beta$-only CARs, transfected Jurkat cells were fixed and stained with cognate target pMHC tetramers in the presence or absence of nonionic detergent for permeabilization (Supplementary Fig. S4). No obvious difference in surface expression or intracellular aggregation among constructs with high and low backgrounds was observed.

To identify the minimal module that is required for epitope binding of V $\beta$-only CAR constructs, CAR vectors were generated where the $\mathrm{C} \beta$ domain is replaced with a CD8 hinge (Supplementary Fig. S5). Peptide-concentration-dependent NFAT signal was observed with these constructs with only modest decrease in sensitivity, indicating that $\mathrm{C} \beta$ is not necessary for the V $\beta$-only binding ability (Supplementary Fig. S5).

Cells transfected with svd TCR did not signal, consistent with the lack of tetramer binding observed in these cells.

svd TCRs are hindered by the V $\alpha$ domain. To further examine surface expression and full TCR complex formation in T cell lines, SUP-T1 cells were transfected with the $\beta$ chains containing the V $\beta$-only domains and a TCR $\alpha$ surrogate chain. SUP-T1 cells are derived from a lymphoma with a chromosomal inversion within the TCR 


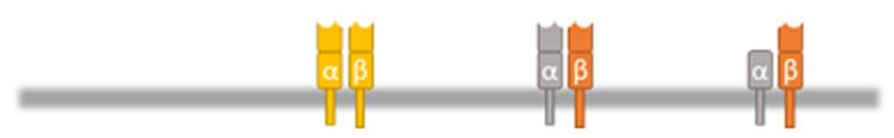

b
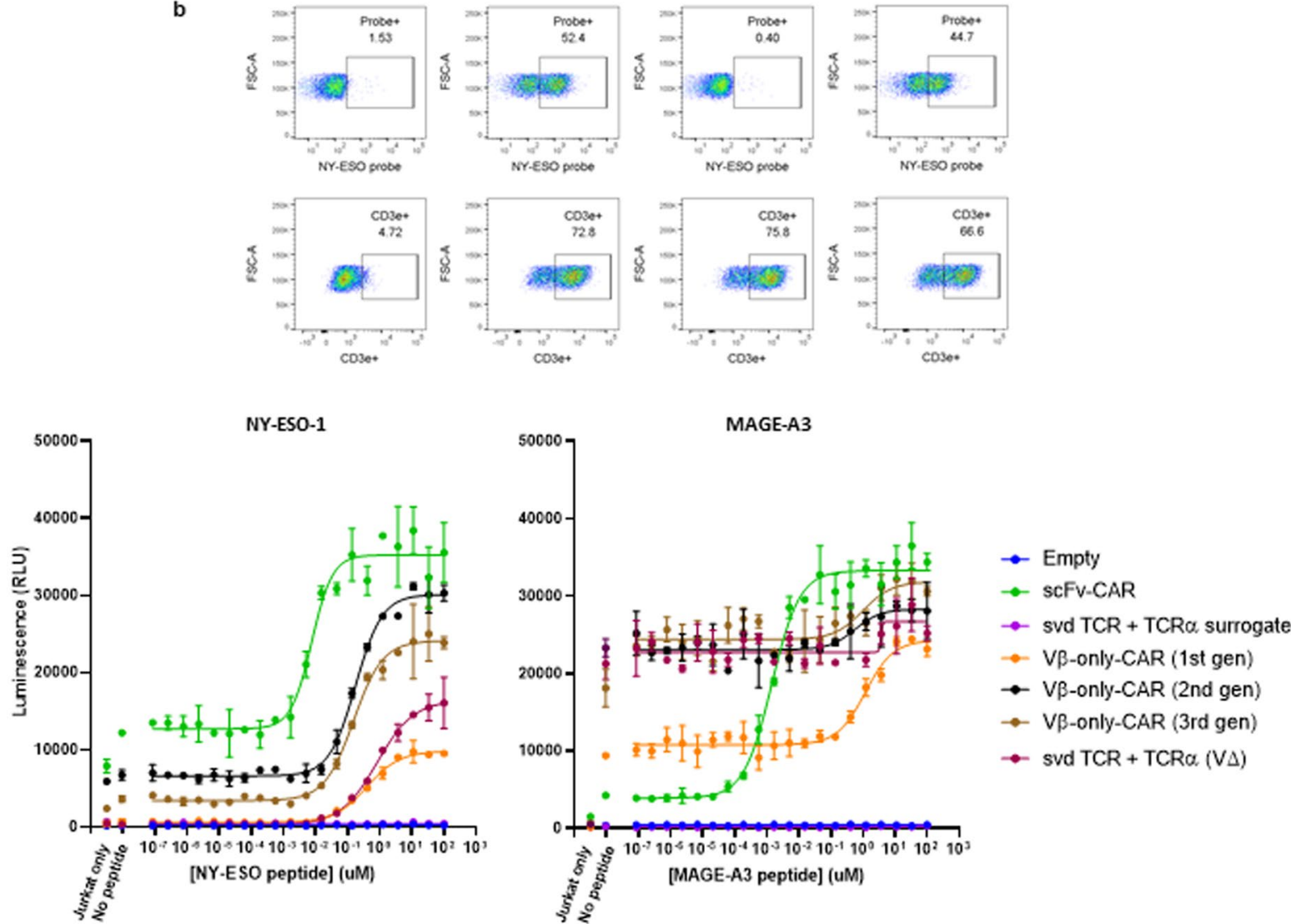

Figure 4. Removal of V $\alpha$ enables svd TCR function. (a) Schematics of the constructs tested. (b) pMHC tetramer binding and TCR complex formation (CD3 $\varepsilon$ surface expression) in transfected SUP-T1 cells. (c) NFAT-luciferase signal of transfected Jurkat cells after $6 \mathrm{hrs}$ of co-culture with NY-ESO-1 (left) or MAGE-A3 (right) peptide-loaded T2 cells. The error bars indicate SD $(n=2)$.

$\alpha$ and $\beta$ gene cluster that prevents surface expression of the endogenous TCR complex. Because TCR $\alpha$ and $\beta$ are not expressed, other subunits of the TCR complex, namely 4 CD3 proteins, are expressed but unable to assemble on the surface ${ }^{20}$. Therefore, $\mathrm{CD} 3 \varepsilon$ staining was used as a readout for expression and pairing of the transfected TCR $\alpha$ and TCR $\beta$ constructs on the cell surface. As previously reported, the NY-ESO-1 control TCR is expressed on the surface of SupT1 cells. Moreover, the V $\beta$-only complex also are positive for CD3E staining, suggesting that the complex integrates the $\beta$ and $\alpha$ subunits. However, only the control TCR showed tetramer binding, consistent with the observation in transfected Jurkat cells (Fig. 4b).

Because we observed pMHC tetramer binding of V $\beta$-only domains in CAR formats but not as a TCR complex, we hypothesized that the TCR V $\alpha$ may hinder the V $\beta$-only domains' ability to bind targets. To test this hypothesis, the V $\alpha$ domain of the $\alpha$ chain was deleted and co-expressed with V $\beta$ chains in SUP-T1 cells. These cells showed tetramer binding, confirming that the $\mathrm{V} \alpha$ indeed hinders epitope binding of $\mathrm{V} \beta$-only domains (Fig. $4 \mathrm{a}, \mathrm{b}$ ). Furthermore, Jurkat cells transfected with the $\beta$ and V $\alpha$-deficient chains revealed T2/peptide-dependent activation, demonstrating that the svd TCRs when their normal $\mathrm{V} \alpha$ partner is removed but the remainder of the alpha chain is retained (Fig. 4c). Despite this functional activity, tetramer binding was not observed by flow cytometry in transfected Jurkat cells, suggesting that the NFAT reporter system is more sensitive than tetramer binding as we have observed previously.

Attempts to trim the C $\alpha$ domain further resulted in complete loss of tetramer binding of the modified TCRs in SUP-T1 cells and corresponding loss of functional activity in Jurkat cells (Supplementary Fig. S6). This result implies that an intact $\mathrm{C} \alpha$ domain is necessary to form a proper surrogate, and a minimal $\alpha$ polypeptide with only a transmembrane domain is not compatible with $\mathrm{V} \beta$-only domain function, probably because of inefficient TCR complex formation absent the $\mathrm{C} \alpha$ domain, likely caused in part by exposed hydrophobic surfaces at the $\mathrm{C} \alpha / \beta$ interface. 
Comparison of signaling sensitivities of $\mathrm{V} \beta$-only domains in CAR and TCR formats. Our system permits direct comparison of the same ligand-binding domains on a CAR and TCR. Interestingly, the sensitivity of the two formats were similar, which suggests that the ligand-binding domain-its affinity and geometry-is the main determinant of sensitivity rather than the details of the signaling mechanism that differ between the CAR and TCR. This observation is consistent with studies where we grafted scFv against the same pMHC targets onto the control NY-ESO-1 TCR, modified to accept the scFvs by removing the V $\alpha$ and $\mathrm{V} \beta$ domains. These hybrid TCRs have sensitivities in Jurkat signaling assays that are nearly identical to the CARs from which they are derived, nearly 100x less sensitive compared to the parental TCR (Supplementary Fig. S7). These observations suggest that, at least in a controlled system where NFAT activation in Jurkat cells is the measure of sensitivity, the ligand-binding domain dictates the signaling sensitivity.

Bispecific V $\beta$-only domains function in CAR and TCR formats. Recent clinical trials have revealed that current adoptive T-cell therapy is susceptible to antigen escape by tumor cells ${ }^{21,22}$. T cells that recognize multiple antigens offer a prospective safeguard against this problem. Bispecific receptors have been explored and developed in the context of CARs ${ }^{23,24}$. However, TCRs with a capacity to target two different antigens have not been reported to our knowledge. Given the small and versatile binding properties of V $\beta$-only domains, we suspected they might function in a more complicated format as a bifunctional CAR or TCR.

To test this idea, bispecific V $\beta$-only CARs targeting NY-ESO- 1 and MAGE-A3 pMHCs were generated in a second-generation CAR architecture with $C \beta$ and the two $V \beta$ domains connected in tandem via a $\left(\mathrm{G}_{4} \mathrm{~S}\right)_{3} \mathrm{GG}$ flexible linker. Jurkat cells transfected with these constructs showed detectable binding of both NY-ESO-1 and MAGE-A3 tetramers by flow cytometry (Fig. 5a). Consistently, in the NFAT-luciferase reporter assay, bispecific CARs functioned in response to both NY-ESO-1- and MAGE-A3-peptide-loaded T2 cells with only modest declines in their individual sensitivities (Fig. 5b). The binding domain positioned further from the membrane (i.e., at the $\mathrm{N}$-terminus) showed a slight decrease in sensitivity while the one directly attached to $\mathrm{C} \beta$ showed no change compared to the monospecific CARs.

To examine if $\mathrm{V} \beta$-only domains could function as bispecific TCRs, we constructed variants in both cis and trans configurations (i.e., in tandem on the $\beta$ chain, or with one $\mathrm{V} \beta$ domain on each chain, $\mathrm{C} \alpha$ and $\mathrm{C} \beta$ (Fig. $5 \mathrm{c}$ ). As a control, V $\beta$ fused on the TCR $\alpha$ chain was also expressed with a TCR $\beta$ chain that lacked a V $\beta$ domain (i.e., $\mathrm{C} \beta$ only). This construct showed no function in the Jurkat NFAT-luciferase reporter assay, indicating that $\mathrm{V} \beta$ domains moved to the $\alpha$-chain abolishes its function (Fig. 5d). Therefore, the trans bispecific svd TCRs showed functional activity only against the pMHC target of the binder fused on the $\beta$-chain (Fig. $5 \mathrm{~d}$ ). Cis bispecific svd TCRs were generated by connecting two $\mathrm{V} \beta$ domains in tandem via a $\left(\mathrm{G}_{4} \mathrm{~S}\right)_{3} \mathrm{GG}$ flexible linker and expressing this construct with a surrogate TCR $\alpha$ chain with the $\mathrm{V} \alpha$ deleted. To our surprise, bifunctional cis svd TCRs with NY-ESO- 1 binder on the N-terminus followed by MAGE-A3 binder N-terminal to C $\beta$ showed both NY-ESO-1 and MAGE-A3 peptide-dependent signaling in Jurkat cells (Fig. 5d). Cis $\mathrm{V} \beta$ domains in the other orientation with the MAGE-A3 binder at the $\mathrm{N}$-terminus also showed functional activity against both target peptides, although the magnitude of the signal (Emax) with MAGE-A3 peptide was reduced. The $\mathrm{EC}_{50}$ in assays with peptides loaded on T2 was similar for both constructs, compared to the sensitivities of monospecific parental versions of the constructs (Supplementary Table S2). We also tested if there was interaction detectable at a functional level between the two pMHC ligands when supplied to Jurkat cell expressing bispecific constructs. Nothing beyond a potentially additive effect was observed using the analytical methods of Bliss and Loewe independence ${ }^{25,26}$.

Primary T cells expressing V $\beta$-only constructs have cytotoxic activity. To evaluate the effect of $\mathrm{V} \beta$-only domain constructs on $\mathrm{T}$ cell activity, primary $\mathrm{T}$ cells were transduced with lentivirus and expression was confirmed by NY-ESO-1 or MAGE-A3 tetramer staining (Fig. 6a). CAR constructs expressed much better than TCR constructs, most likely due to the mispairing of the introduced TCR chains to endogenous TCR chains. Transduced T cells were used in an IncuCyte cell killing assay that enables visualization of target and effector cells by microscopy at $37^{\circ} \mathrm{C}$ over time. A375 cells that stably express nuclear locating GFP were loaded with $10 \mu \mathrm{M}$ NY-ESO-1 or MAGE-A3 peptides and co-cultured with transduced T cells at 1:1 ratios. T cell number was adjusted according to the transduction percentage measured by tetramer staining.

For NY-ESO-1 binders, T cells expressing the benchmark TCR showed the most potent cytotoxic activity, followed by $\mathrm{T}$ cells expressing the scFv-CAR, and the VB-only domain constructs in CAR and TCR formats which had similar killing activities (Fig. 6b). IFN $\gamma$ measured in the supernatant of the co-culture at 24 hours showed a similar trend (Fig. 6c). K562 cells that overexpress single chain NY-ESO-1- $\beta_{2}$ m-HLA-A2 trimer $^{27}$ and GFP were also used as target cells in the real-time killing assay. In this situation where the antigen is presented abundantly, all 4 NY-ESO-1-targeted constructs showed similar killing activities (Supplementary Fig. S8).

$\mathrm{T}$ cells expressing the MAGE-A3 benchmark TCR and scFv-CARs only showed mild cytotoxic activities while the V $\beta$-only-CAR and svd TCR triggered more robust killing (Fig. 6b). However, these V $\beta$-only-CAR and svd TCR cells also showed weak cytotoxicity toward K562 cells without any MAGE-A3 peptide (Supplementary Fig. S8), suggesting that these constructs likely trigger ligand-independent apoptosis of target cells. This is consistent with the high background NFAT signal observed in Jurkat cells transfected with the MAGE-A3 Vß-only-CAR and svd TCR (Fig. 4c).

\section{Discussion}

We have created $\mathrm{V} \beta$-only domains that express, specifically recognize cognate $\mathrm{pMHC}$ ligands, and function robustly in Jurkat and primary T cells. The generality of this effect is suggested by the isolation and characterization of multiple binders against two different pMHC targets. 
a
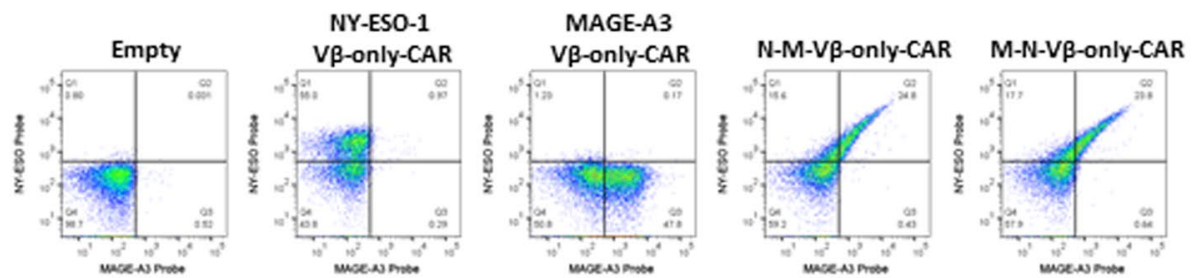

b

NY-ESO-1
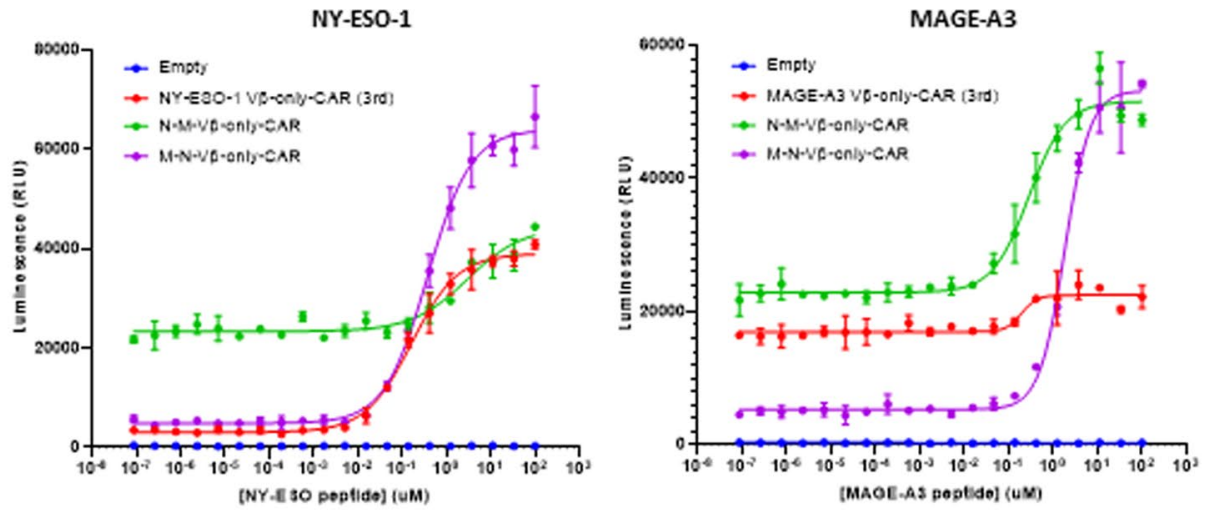

c
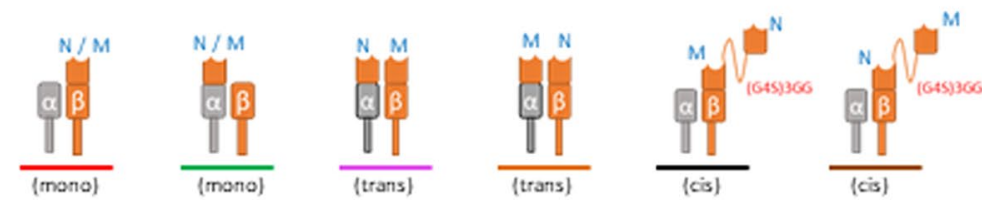

d
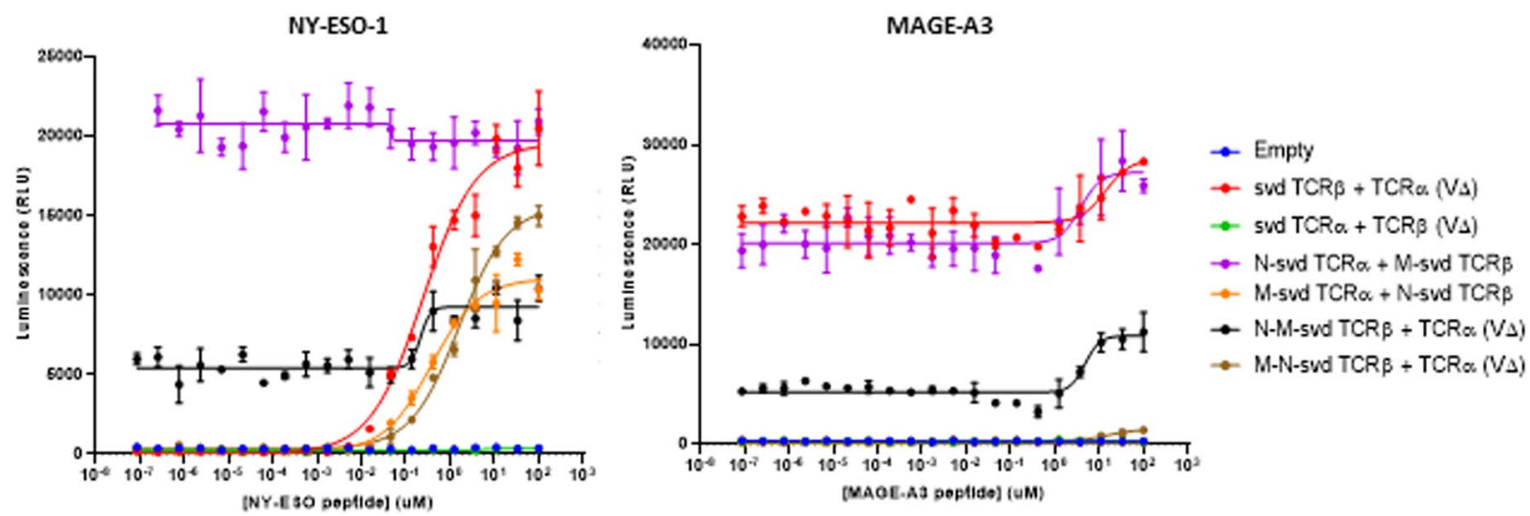

Figure 5. Bifunctional CARs and TCRs with two ligand binding domains. (a) FACS plot of transfected Jurkat cells stained for binding to NY-ESO-1 pMHC tetramer ( $y$-axis) and MAGE-A3 pMHC tetramer (x-axis). M refers to MAGE-A3 and N refers to NY-ESO-1. (b) NFAT-luciferase signal of transfected Jurkat cells after $6 \mathrm{hrs}$ of coculture with NY-ESO-1 (left) and MAGE-A3 (right) peptide-loaded T2 cells. The error bars indicate SD $(\mathrm{n}=2)$. (c) Schematics of the constructs tested. M refers to MAGE-A3 and N refers to NY-ESO-1. (d) Same as (b).

In a TCR format, the $\beta$ chain utilize a surrogate $\alpha$ chain that lacks a $V \alpha$ segment and forms activation-competent TCRs complexed with the six CD3 subunits. We presume the requirement for a surrogate $\alpha$ subunit relates to the formation of a complex in the membrane with other CD3 subunits that comprise a functional TCR. Sequential $\mathrm{N}$-terminal deletions demonstrate that the globular $\mathrm{C} \alpha$ domain is necessary for full activity of the svd TCR. In contrast, the presence of a V $\alpha$ domain on the $\alpha$ subunit, opposite the single V $\beta$ polypeptide, has no impact on surface expression, but interferes with ligand-binding, presumably via steric hindrance of the pMHC ligand to its cognate $\mathrm{V} \beta$ binding site.

Selectivity of the svd TCRs compares favorably with other TCRs and antibodies that bind pMHCs. They retain the HLA restriction for which they were selected. Thus, the fact that evolution has favored a two-domain ligand-binding structure does not necessarily preclude the creation of selective single-domain binders, a feature that also applies to the antibody gene family ${ }^{28}$. Rather, it may be that a repertoire composed of two chains generates necessary diversity through the agency of combinatorial association. 

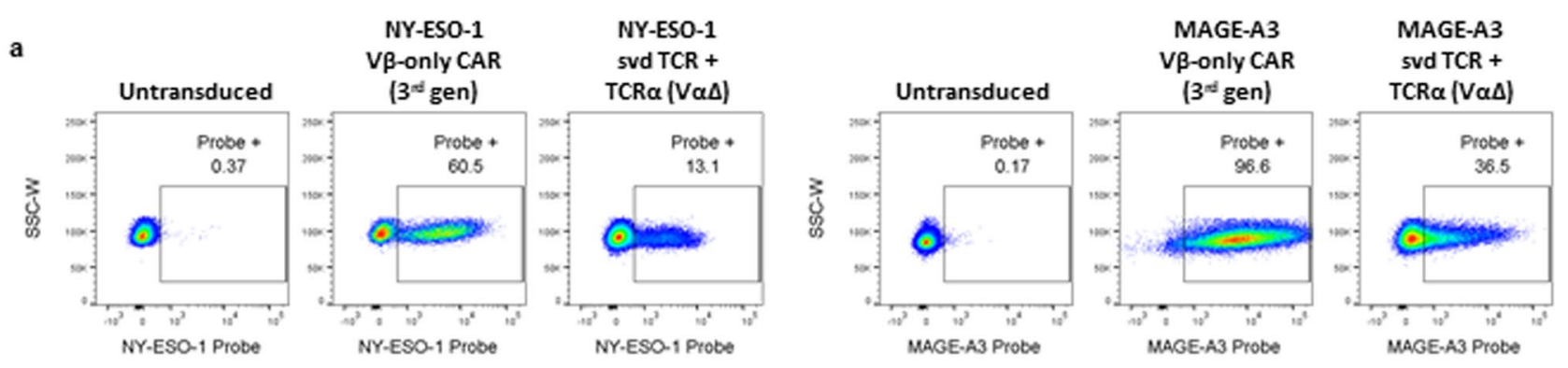

b

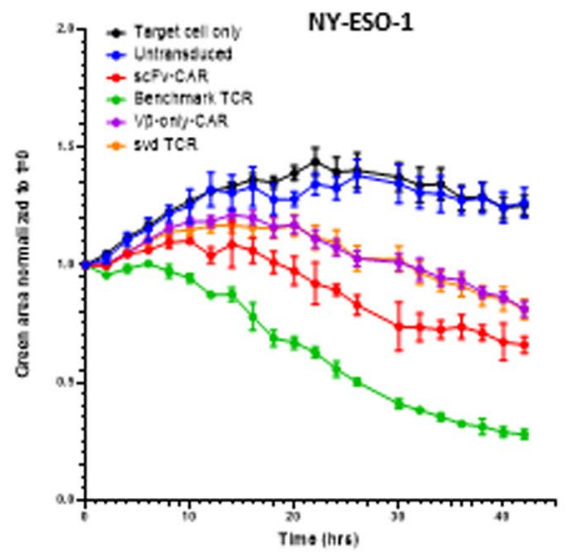

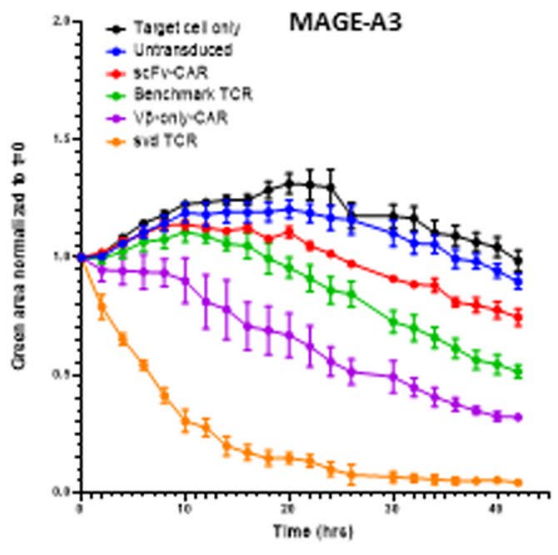

c

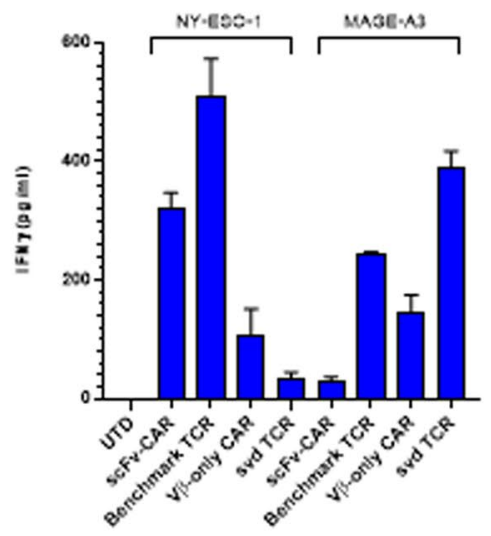

Figure 6. V $\beta$-only-CARs and svd TCRs expressed in primary T-cells show cytotoxicity and release cytokines. (a) Primary T cells transduced with indicated constructs stained with NY-ESO-1 or MAGE-A3 probes. (b) A375 cells expressing nuclear locating GFP loaded with $10 \mu \mathrm{M}$ NY-ESO-1 (left) or MAGE-A3 (right) peptides were co-cultured with T cells transduced with NY-ESO-1 (left) or MAGE-A3 (right) binding constructs at 1:1 ratio and imaged in IncuCyte for 42 hours. Ratio of total green fluorescent area at each time point divided by time zero value is plotted. The error bar indicates $\mathrm{SD}(\mathrm{n}=2)$. (c) IFN $\gamma$ measured by CBA assay with supernatants from the 24 hour time-point of the co-cultures in $(\mathbf{b})$. The error bars indicate $\mathrm{SD}(\mathrm{n}=2)$.

In addition, the ligand-binding module can be grafted onto CAR signaling constructs where it functions independent of a TCR $\alpha$ chain. Both ligand-binding domains examined most closely (one NY-ESO-1 and one MAGE-A3 binder) are compatible with a variety of CAR formats that incorporate different CAR components (Figs. 1,3). Interestingly, the CAR versions of the $\mathrm{V} \beta$-only ligand-binding domains have similar sensitivity to their TCR cousins. This result suggests that the properties of the ligand-binding domain determine functional sensitivity, regardless of the format; i.e., it is not the detailed structure of the signaling components, but rather, the binding properties of the receptor that dictate sensitivity, at least in the Jurkat NFAT-activation assay.

This conclusion is strengthened by the observation that $\mathrm{scFv}$ sequences derived from antibodies generated against the same pMHC targets (NY-ESO-1 and MAGE-A3), when grafted onto TCR subunits, yield hybrid TCRs that are nearly identical in sensitivity to CAR constructs that harbor the same scFvs (Supplementary Fig. S6). Thus, with two different pMHC targets and two independent origins of ligand-binding domains, one pair derived from TCRs and the other from antibodies, the sensitivity uniformly tracks with the ligand-binding domain, not the type of signaling molecule. These results again suggest that TCRs may acquire their striking sensitivity not by their highly evolved, multi-subunit mechanism, but rather through the process of selection in the body. It is possible that both $\alpha$ and $\beta$ chains need to interact with the cognate MHC to deliver a sensitive signal. However, analysis of previously reported TCR-pMHC structures and amino acid sequences suggest that there are multiple instances where CDR3 $\alpha$ makes no contact with the target $p M H C$ whereas CDR3 3 contact is always present ${ }^{29}$. For TCRs isolated and studied in vitro by investigators, their extraordinary potency may be further explained by additional functional selection through growth on antigen-positive substrates or picking rare antigen-responsive $\mathrm{T}$ cells clones among hundreds of thousands of $\mathrm{T}$ cells ${ }^{16,17}$.

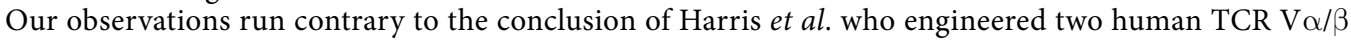
pairs directed at either a WT- 1 or MART- 1 pMHC $^{30}$. When these affinity-matured binders were tested as either reconstructed TCRs or CARs, the TCR versions were 10-100x more sensitive in both cases. The reasons for the discrepancy remain to be established, but include the possibilities that: (i) The $\mathrm{V} \alpha / \beta$ interaction is a critical part of the TCR signaling mechanism, and therefore the Vß-only TCR is crippled with respect to TCR sensitivity because it lacks Vo; (ii) IL-2 expression used by Harris et al. as a readout favors the TCR signaling mechanism compared to NFAT activation measured in the Jurkat assay conducted by us; (iii) their use of a murine T cell hybridoma line and murine primary $\mathrm{T}$ cells as effectors with a human $\mathrm{T} 2$ lymphoid line as stimulus creates an interspecies 
activation scenario that somehow biases sensitivity toward TCRs; or, (iii) optimal TCR function follows from TCRs that are subjected to strong functional selection; if they originate from physical binding selections like the ones studied here, they lack the extraordinary sensitivity characteristic of some functionally-selected TCRs and, instead, display sensitivities equivalent to their CAR counterpart.

Signaling behavior of the V $\beta$-only CARs and svd TCRs are similar in many respect to TCRs regarding the shape of the dose/response curve (Fig. 4). However, they are less sensitive than the most highly selected or optimized TCRs, which are typically obtained from patients' functionally selected T cells, or otherwise optimized for activity $^{16,17}$, including the benchmark NY-ESO-1 TCR used in our experiments. The MAGE-A3-targeting V $\beta$-only module showed high background (tonic signal) and a narrow activation window in Jurkat cells (Figs. 3, 4c). Note that V $\beta$-only modules used in this study are not optimized. Sensitivity, tonic signaling, and activation windows are properties that can be optimized by screening larger numbers of variants (unpublished data). For example, the other three idiotypes (ID \#1, 2, 4) of MAGE-A3 Vß-only modules show much lower tonic signal than ID \#5 (Supplementary Fig. S3), which we initially chose for further study due to its strongest tetramer binding ability (Supplementary Fig. S1). Based on a broad range of studies with TCRs, we believe efforts to optimize binding of V $\beta$-only-modules would produce more potent receptors ${ }^{31-35}$. In other words, there is nothing obvious in a $\mathrm{V} \beta$-only ligand binding domain that precludes it from mimicking a natural TCR in all its fundamental attributes. However, we acknowledge that further validation and optimization will be needed to prove the utility of this technology beyond its academic interest.

Considerable effort has been directed at antibodies to engineer single-variable domains that avoid the requirement for a second chain. The goal is to make the ligand-binding function smaller and more easily shuttled from one molecular setting to another. The existence of natural single-domain antibodies, notably from the family Camelidae (including camels and llamas) and class Chondrichthyes (including sharks), has pointed the way toward engineering similar fully human domains. These small phylogenetically distant single-domain antibodies represent a significant variation of the classic immunoglobulin fold in which the surface that is typically involved in the interaction with a light chain variable domain is altered and covered by an extension of the CDR3 loop, resulting in a more compact, convex globular structure ${ }^{36-38}$. Efforts to engineer antibody structures to produce single-domain (e.g., heavy-chain only) variable domains, have required the introduction of substantial amino acid variation and structural change that create molecules more similar to the camelid single-domain molecules, compared to the two-chain structures from which they derive $\mathrm{e}^{39,40}$.

Although we have not elucidated the atomic structure of the V $\beta$-only domains described here, we strongly suspect that they also involve considerable deviation from the two-chain TCR. Notably, all the V $\beta$-only domains identified use the V $\beta 5-8$ variable gene segment. This bias is not explained by a skewed TCR input library because TRB-5-8, the V gene segment involved, was present at roughly the expected frequency in the HuTARG ${ }^{\mathrm{TM}}$ library prior to induced TCR rearrangement ( $\sim 1 \%$ of total V segments; data not shown). Its frequency was increased about 6-fold among cells that express surface TCRs, consistent with the view that it may more readily form stable globular domains. This possibility is supported by modeling (Fig. 2).

V $\beta$-only domains provide an opportunity to create bifunctional TCR ligand-binding domains that are smaller and more portable-potentially very useful in the context of CAR-T and TCR-T engineering. In particular, investigators have created bifunctional CARs that incorporate scFvs in tandem to direct engineered T cells at two separate antigens; e.g., CD19 and CD20 $0^{23,24}$. The hypothesis is that such dual-targeted receptors will pose additional obstacles for tumor relapse, as the tumor must escape two rather than one T-cell targeting mechanisms. Such approaches have also been proposed for CARs, but the technical route toward TCR bifunctionality is less clear.

We have taken a significant step toward this goal by demonstrating that $\mathrm{V} \beta$-only domains can be linked in tandem with only modest decline in their individual sensitivities to generate small, bifunctional single-V $\beta$-based CARs and TCRs. In principle, deployment of suitable TCR V $\beta$ s directed at different pMHCs permits construction of hybrid molecules activated by both pMHCs in a single engineered T cell, without either the problems associated with mispairing of multiple transferred subunits or the size limits imposed by current viral gene transfer vectors.

Interestingly, the bifunctional TCRs display improved functional selectivity compared to the individual binding domains tested on their own (Fig. 5b,d). Particularly the MAGE-A3 svd TCR exhibits some evidence of off-target activation when exposed to HLA-A2-positive T2 cells. However, in the bifunctional format this background nearly disappears. Though we do not have a simple explanation for this change, it suggests that the bifunctional format is at least compatible with production of stable, well-behaved surface receptors that bind ligands specifically.

\section{Methods}

Cell culture. HEK-293T (ATCC CRL-3216 ${ }^{\mathrm{TM}}$ ), Jurkat clone E6-1 (ATCC TIB-152 ${ }^{\mathrm{TM}}$ ), T2 (ATCC CRL-1992 ${ }^{\mathrm{TM}}$ ), SUP-T1 (ATCC CRL-1942 ${ }^{\mathrm{TM}}$ ), A375 (ATCC CLR-1619 ${ }^{\mathrm{TM}}$ ) and K562 (ATCC CCL-243 ${ }^{\mathrm{TM}}$ ) cell lines were used in this study. HEK-293T and A375 cells were maintained in DMEM supplemented with $10 \%$ fetal bovine serum (FBS) and 1\% penicillin/streptomycin. Jurkat and SUP-T1 cells were maintained in RPMI supplemented with $10 \%$ heat-inactivated FBS and $1 \%$ penicillin/streptomycin. T2 cells were maintained in IMDM supplemented with $20 \%$ FBS and 1\% penicillin/streptomycin. K562 cells engineered to stably express single chain NY-ESO-1$\beta_{2}$ m-HLA-A2 trimer ${ }^{27}$ and GFP were generated and maintained in RPMI supplemented with $10 \%$ heat-inactivated FBS and $1 \%$ penicillin/streptomycin. Cells were incubated at $37^{\circ} \mathrm{C}$ in $5 \% \mathrm{CO}_{2}$.

Plasmid construction. All constructs, except for the recovered V $\beta$-only pool from the HuTARG ${ }^{\mathrm{TM}}$ platform (Patent WO2017/091905), were generated by Golden Gate assembly into the pLenti backbone, which has an EF1 $\alpha$ promoter. NY-ESO-1 and MAGE-A3 scFvs on the control CARs were obtained from binder screens using the HuTARG $^{\mathrm{TM}}$ platform. Sequences for the control NY-ESO-1 and MAGE-A3 TCRs were obtained from patents US8143376B2 and W02012054825A1, respectively. 
HuTARG ${ }^{\mathrm{TM}}$. See patent WO2017/091905 for details. Briefly, HEK293.2sus (ATTC CRL15733 ${ }^{\mathrm{TM}}$ ) cells were engineered to contain a library of all combinations of unrearranged V, D and J segments of the $\beta$ chain TCR locus. Because the host cells are constructed to contain a single loxP integration site, each cell post transfection of the library contains at most a single integrated copy of one of the roughly 1,800 combinations of V-D-J $(64 \times 2 \times 14)$ gene segments, each segment is flanked by a recombination signal sequence (RSS). When triggered to rearrange by addition of an exogenous inducer that activates RAG1 expression, the RSSs mediate intramolecular site-specific recombination such that the population of cells produce a large repertoire of TCR $\beta$ chains that are further subjected to drug selection for in-frame recombinants; the TCR $\beta$ chain is expressed as a fusion with the puromycin resistance gene. The cells also contain a surrogate single alpha chain for pairing and surface expression.

Transfection. Transient transfections of T-cell lines were performed using the Neon Transfection System (Thermo Fisher Scientific, Cat. No. MPK5000) according to the manufacturer's instructions. Cells were pulsed three times at $1,500 \mathrm{~V}$ and a width of $30 \mathrm{msec}$. Transient transfection of HEK-293T cells were performed using the Fugene HD transfection method (Promega, Cat. No. E2311) according to the manufacturer's instructions.

FACS analysis. 18 hours post-transfection, cells were harvested and washed three times with FACS buffer $(\mathrm{PBS}+0.1 \% \mathrm{BSA})$. The cells were incubated with TCR $\beta \mathrm{F} 1$ (8A3) antibody conjugated with PE-Cy7 (Life Technologies, Cat. No. 25576641), CD3 epsilon antibody conjugated with FITC, Alexa Fluor 647-Streptavidin (Jackson, Cat. No. 016-600-084)-labeled MHC Class I A*02:01 SLLMWITQV (NY-ESO), or Alexa Fluor 647-Streptavidin-labeled MHC Class I A*02:01 FLWGPRALV (MAGE-A3) for 1 hour at $4^{\circ} \mathrm{C}$. Cells were then washed twice, stained with DAPI or the near-IR dead cell stain kit reagents (Thermo Fisher, Cat. No. L34976), and resuspended in FACS buffer for flow cytometer analysis. Data acquisition used the BD Canto instrument and software. Data were analyzed using Flowjo software.

Homology model and hydrophobic patch calculation. 3D structural models were generated in Prime homology modeling suite (Schrödinger, LLC) using the crystal structure of the MAGE-A3 TCR (TRAV21/ TRBV5-1 family; PDBID: 5BRZ) as the template. All structures were prepared using the Schrödinger Protein Preparation Wizard ${ }^{41}$ before further calculations.

Hydrophobic patches on the surface of the homology model and crystal structures were calculated and visualized using the Protein Surface Analysis tool as implemented in Bioluminate (Schrödinger, LLC) ${ }^{42}$.

Molecular dynamics simulation. The system was solvated using the Desmond System Builder (Schrödinger, LLC). Sodium ions were added to neutralize the system. The OPLS3e force field was utilized for all calculations ${ }^{43}$. The system equilibrated using the relaxation protocol as implemented in the Schrödinger package. Molecular Dynamics simulations were performed in the NPT ensemble at $300 \mathrm{~K}$ and 1 atm pressure using Desmond (D.E. Shaw Research). The reversible reference system propagation algorithm (RESPA) multiple time step approach was used with a time step of 2 psec and long-ranged electrostatic interactions were computed every 6 psec. ${ }^{44}$. Van der Waals and short-range electrostatic interactions were cut off at $9 \mathrm{~A}^{\circ}$ and smooth particle mesh Ewald (PME) method ${ }^{45}$ was employed for calculation of long range electrostatic interactions. The temperature was controlled using a Nose-Hoover chain thermostat ${ }^{46}$ and the pressure was controlled using the Martyna-Tobias-Klein barostat ${ }^{47}$. Three simulations of $100 \mathrm{~ns}$ each were performed using the last frame as the initial coordinates on a Quadro P5000 GPU card with coordinates saved every 1.2 ps for subsequent analysis.

NFAT Luciferase assay. NY-ESO-1 peptide (SLLMWITQV), a variant of the native peptide where cysteine at position 9 is mutated to Valine ${ }^{48}$, and MAGE-A3 peptide (FLWGPRALV) were synthesized by Genscript (Piscataway, NJ). Target peptides were serially diluted 3-fold starting at $100 \mu \mathrm{M}$ and loaded onto 10,000 T2 cells in RPMI plus $1 \%$ BSA and $0.1 \%$ penicillin/streptomycin. Eighteen hours later, 12,000 transfected Jurkat cells encoding a NFAT-luciferase reporter were resuspended in RPMI/10\% FBS/0.1\% penicillin/streptomycin and added to the peptide-loaded T2 cells. The co-culture was incubated for 6 hours at $37^{\circ} \mathrm{C}$. One-step luciferase assay system (BPS Cat. No. 60690) was used according to manufacturer's instructions to read luminescence on a microplate luminometer at $100 \mathrm{~ms}$. Each experiment was done in duplicate.

Primary T-cell transduction and activation. Peripheral blood mononuclear cells (PBMCs) and lentivirus were obtained from Alstem. PBMCs were activated with human T-cell TransAct ${ }^{\mathrm{TM}}$ according to manufacturer's instructions in X-vivo media. For the transduction, lentivirus was added directly to the cells at a MOI of 10. Transduced primary T-cells were expanded in X-vivo medium with $1 \%$ human serum, $0.1 \%$ penicillin/streptomycin, $10 \mathrm{ng} / \mathrm{ml} \mathrm{IL-15}$, and $10 \mathrm{ng} / \mathrm{ml} \mathrm{IL-21}$. Cells were quiesced for 48 hours without stimulation or cytokines prior to assays.

Cytotoxicity and IFN $\gamma$ assay. A375 cells were transfected with nuclear locating GFP (IncuCyte NucLight Green) and selected for stable integration. Five thousand A375 cells were seeded with $10 \mu \mathrm{M}$ of NY-ESO-1 or MAGE-A3 peptides. After 18 hours, cells were treated with $10 \mu \mathrm{g} / \mathrm{ml}$ of mitomycin C for 1 hour and washed three times. Five thousand transduced T-cells (adjusted for transduction percentage measured by tetramer staining) were added and monitored using the IncuCyte S3 live-cell analysis system (Essen Bioscience) at $37{ }^{\circ} \mathrm{C}$ and $5 \%$ $\mathrm{CO}_{2}$. Images were captured every two hours for 42 hours using a $10 \mathrm{X}$ objective. Green area at each time point was normalized to time zero to measure loss of live target cells. Experiments were done in duplicate.

Supernatants were harvested at the 24 hour time-point of the cytotoxicity assay co-culture. IFN $\gamma$ levels were measured using the BD Cytometric bead array system according to manufacturer's instruction. 
Statistical analysis. For all data comparisons, the Student's t test was performed using GraphPad Prism software. $\mathrm{EC}_{50}$ values were obtained by fitting the peptide concentration-dependent NFAT-Luciferase readout by nonlinear regression.

Received: 23 August 2019; Accepted: 31 October 2019; Published online: 21 November 2019

\section{References}

1. Neelapu, S. S. et al. Axicabtagene Ciloleucel CAR T-Cell Therapy in Refractory Large B-Cell Lymphoma. N Engl J Med 377, 2531-2544 (2017).

2. Maude, S. L. et al. Tisagenlecleucel in Children and Young Adults with B-Cell Lymphoblastic Leukemia. N Engl J Med 378, 439-448 (2018).

3. Wieczorek, M. et al. Major Histocompatibility Complex (MHC) Class I and MHC Class II Proteins: Conformational Plasticity in Antigen Presentation. Front Immunol 8, 292 (2017).

4. Wright, C. A., Kozik, P., Zacharias, M. \& Springer, S. Tapasin and other chaperones: models of the MHC class I loading complex. Biol Chem 385, 763-778 (2004).

5. Alcover, A., Alarcon, B. \& Di Bartolo, V. Cell Biology of T Cell Receptor Expression and Regulation. Annu Rev Immunol 36, 103-125 (2018).

6. Huppa, J. B. et al. TCR-peptide-MHC interactions in situ show accelerated kinetics and increased affinity. Nature 463, 963-967 (2010).

7. Krogsgaard, M. et al. Agonist/endogenous peptide-MHC heterodimers drive T cell activation and sensitivity. Nature 434, 238-243 (2005).

8. Criscitiello, M. F., Saltis, M. \& Flajnik, M. F. An evolutionarily mobile antigen receptor variable region gene: doubly rearranging NAR-TcR genes in sharks. Proc Natl Acad Sci USA 103, 5036-5041 (2006).

9. Greenberg, A. S. et al. A new antigen receptor gene family that undergoes rearrangement and extensive somatic diversification in sharks. Nature 374, 168-173 (1995).

10. Rouet, R., Dudgeon, K., Christie, M., Langley, D. \& Christ, D. Fully Human VH Single Domains That Rival the Stability and Cleft Recognition of Camelid Antibodies. J Biol Chem 290, 11905-11917 (2015).

11. Dottorini, T., Vaughan, C. K., Walsh, M. A., LoSurdo, P. \& Sollazzo, M. Crystal structure of a human VH: requirements for maintaining a monomeric fragment. Biochemistry 43, 622-628 (2004).

12. Krah, S. et al. Single-domain antibodies for biomedical applications. Immunopharmacol Immunotoxicol 38, 21-28 (2016).

13. Gonzalez-Sapienza, G., Rossotti, M. A. \& Tabares-da Rosa, S. Single-Domain Antibodies As Versatile Affinity Reagents for Analytical and Diagnostic Applications. Front Immunol 8, 977 (2017).

14. Hou, X. et al. High Throughput Sequencing of T Cell Antigen Receptors Reveals a Conserved TCR Repertoire. Medicine (Baltimore) 95, e2839 (2016).

15. Invitrogen TCR V beta F1 Monoclonal Antibody (8A3), PE-Cyanine7, eBioscience ${ }^{\mathrm{TM}}$. Catalog \# 25-5766-42 Data Sheet.

16. Robbins, P. F. et al. Single and dual amino acid substitutions in TCR CDRs can enhance antigen-specific T cell functions. J Immunol 180, 6116-6131 (2008).

17. Chinnasamy, N. et al. A TCR targeting the HLA-A*0201-restricted epitope of MAGE-A3 recognizes multiple epitopes of the MAGE-A antigen superfamily in several types of cancer. J Immunol 186, 685-696 (2011).

18. Raman, M. C. et al. Direct molecular mimicry enables off-target cardiovascular toxicity by an enhanced affinity TCR designed for cancer immunotherapy. Sci Rep 6, 18851 (2016).

19. Chan, K. F. et al. Divergent T-cell receptor recognition modes of a HLA-I restricted extended tumour-associated peptide. Nat Commun 9, 1026 (2018).

20. Morgan, R. A. et al. High efficiency TCR gene transfer into primary human lymphocytes affords avid recognition of melanoma tumor antigen glycoprotein 100 and does not alter the recognition of autologous melanoma antigens. J Immunol 171, 3287-3295 (2003).

21. Grupp, S. A. et al. Chimeric antigen receptor-modified T cells for acute lymphoid leukemia. N Engl J Med 368, 1509-1518 (2013).

22. Maude, S. L. et al. Chimeric antigen receptor T cells for sustained remissions in leukemia. N Engl J Med 371, 1507-1517 (2014).

23. Grada, Z. et al. TanCAR: A Novel Bispecific Chimeric Antigen Receptor for Cancer Immunotherapy. Mol Ther Nucleic Acids 2, e105 (2013).

24. Zah, E., Lin, M. Y., Silva-Benedict, A., Jensen, M. C. \& Chen, Y. Y. T Cells Expressing CD19/CD20 Bispecific Chimeric Antigen Receptors Prevent Antigen Escape by Malignant B Cells. Cancer Immunol Res 4, 498-508 (2016).

25. Bliss, C. I. The toxicity of poisons applied jointly. Annals of Applied Biology 26, 585-615 (1939).

26. Loewe, $S$. The problem of synergism and antagonism of combined drugs. Arzneimittelforschung 3, 285-290 (1953).

27. Hansen, T., Yu, Y. Y. \& Fremont, D. H. Preparation of stable single-chain trimers engineered with peptide, beta2 microglobulin, and MHC heavy chain. Curr Protoc Immunol Chapter 17, Unit17 15 (2009).

28. Arbabi-Ghahroudi, M. Camelid Single-Domain Antibodies: Historical Perspective and Future Outlook. Front Immunol 8, 1589 (2017).

29. Glanville, J. et al. Identifying specificity groups in the T cell receptor repertoire. Nature 547, 94-98 (2017).

30. Harris, D. T. et al. Comparison of T Cell Activities Mediated by Human TCRs and CARs That Use the Same Recognition Domains. J Immunol 200, 1088-1100 (2018)

31. Cohen, C. J. et al. Enhanced antitumor activity of T cells engineered to express T-cell receptors with a second disulfide bond. Cancer Res 67, 3898-3903 (2007).

32. Cohen, C. J., Zhao, Y., Zheng, Z., Rosenberg, S. A. \& Morgan, R. A. Enhanced antitumor activity of murine-human hybrid T-cell receptor (TCR) in human lymphocytes is associated with improved pairing and TCR/CD3 stability. Cancer Res 66, 8878-8886 (2006).

33. Kuball, J. et al. Facilitating matched pairing and expression of TCR chains introduced into human T cells. Blood 109, 2331-2338 (2007).

34. Sommermeyer, D. \& Uckert, W. Minimal amino acid exchange in human TCR constant regions fosters improved function of TCR gene-modified T cells. J Immunol 184, 6223-6231 (2010).

35. Kieke, M. C. et al. High affinity T cell receptors from yeast display libraries block T cell activation by superantigens. J Mol Biol 307, 1305-1315 (2001).

36. Bond, C. J., Marsters, J. C. \& Sidhu, S. S. Contributions of CDR3 to V H H domain stability and the design of monobody scaffolds for naive antibody libraries. J Mol Biol 332, 643-655 (2003).

37. Stanfield, R. L., Dooley, H., Flajnik, M. F. \& Wilson, I. A. Crystal structure of a shark single-domain antibody V region in complex with lysozyme. Science 305, 1770-1773 (2004).

38. Desmyter, A. et al. Crystal structure of a camel single-domain VH antibody fragment in complex with lysozyme. Nat Struct Biol 3, 803-811 (1996). 
39. Desmyter, A., Decanniere, K., Muyldermans, S. \& Wyns, L. Antigen specificity and high affinity binding provided by one single loop of a camel single-domain antibody. J Biol Chem 276, 26285-26290 (2001).

40. Davies, J. \& Riechmann, L. 'Camelising' human antibody fragments: NMR studies on VH domains. FEBS Lett 339, 285-290 (1994).

41. Sastry, G. M., Adzhigirey, M., Day, T., Annabhimoju, R. \& Sherman, W. Protein and ligand preparation: parameters, protocols, and influence on virtual screening enrichments. J Comput Aided Mol Des 27, 221-234 (2013).

42. Sankar, K., Krystek, S. R. Jr., Carl, S. M., Day, T. \& Maier, J. K. X. AggScore: Prediction of aggregation-prone regions in proteins based on the distribution of surface patches. Proteins 86, 1147-1156 (2018).

43. Harder, E. et al. OPLS3: A Force Field Providing Broad Coverage of Drug-like Small Molecules and Proteins. J Chem Theory Comput 12, 281-296 (2016)

44. Tuckerman, M. \& Berne, B. J. Reversible multiple time scale molecular dynamics. The Journal of Chemical Physics 97 (1992).

45. Essmann, U. et al. A Smooth Particle Mesh Ewald Method, Vol. 103. (1995).

46. Martyna, G. J., Klein, M. L. \& Tuckerman, M. Nosé-Hoover chains: The canonical ensemble via continuous dynamics. The Journal of Chemical Physics 97, 2635-2643 (1992).

47. Martyna, G. J., Tobias, D. J. \& Klein, M. L. Constant pressure molecular dynamics algorithms. The Journal of Chemical Physics 101, 4177-4189 (1994).

48. Chen, J. L. et al. Identification of NY-ESO-1 peptide analogues capable of improved stimulation of tumor-reactive CTL. J Immunol 165, 948-955 (2000).

\section{Acknowledgements}

We are grateful to M. Daris for his skillful construction of CAR and TCR vectors, and thank Drs. A. Hamburger, J. Yi, J. Johnston and M. Davis for useful discussions and comments on the manuscript.

\section{Author contributions}

A.K. conceived and supervised the overall project. J.K. and M.G. supervised the initial identification of V $\beta$-only domains. J.O., M.M. and M.L.M. performed the experiments. D.T.W. performed the modeling analysis for Fig. 2. A.L., F.C. and C.P. performed the experiments for the initial identification of V $\beta$-only domains. A.K. and J.O. wrote the manuscript with the help of all other authors.

\section{Competing interests}

A patent has been filed relating to some of the data presented. The authors are employees of A2 Biotherapeutics, Inc. or Innovative Targeting Solutions, Inc.

\section{Additional information}

Supplementary information is available for this paper at https://doi.org/10.1038/s41598-019-53756-4.

Correspondence and requests for materials should be addressed to A.K.

Reprints and permissions information is available at www.nature.com/reprints.

Publisher's note Springer Nature remains neutral with regard to jurisdictional claims in published maps and institutional affiliations.

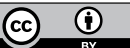

Open Access This article is licensed under a Creative Commons Attribution 4.0 International License, which permits use, sharing, adaptation, distribution and reproduction in any medium or format, as long as you give appropriate credit to the original author(s) and the source, provide a link to the Creative Commons license, and indicate if changes were made. The images or other third party material in this article are included in the article's Creative Commons license, unless indicated otherwise in a credit line to the material. If material is not included in the article's Creative Commons license and your intended use is not permitted by statutory regulation or exceeds the permitted use, you will need to obtain permission directly from the copyright holder. To view a copy of this license, visit http://creativecommons.org/licenses/by/4.0/.

(C) The Author(s) 2019 